\title{
Aptamer-based fluorescence sensor for rapid detection of potassium ions in urine $\dagger$
}

\author{
Chih-Ching Huang and Huan-Tsung Chang*
}

Received (in Cambridge, UK) 4th December 2007, Accepted 8th January 2008

First published as an Advance Article on the web 24th January 2008

DOI: $10.1039 / \mathbf{b} 718752 a$

\begin{abstract}
We unveil a new homogeneous assay-using OliGreen and an ATP-binding aptamer-for the highly selective and sensitive detection of potassium ions.
\end{abstract}

Potassium ions $\left(\mathrm{K}^{+}\right)$are involved in many biological functions, including nerve transmission, regulation of blood pressure and $\mathrm{pH}$, enzyme activation, and the formation of collagen or elastin. ${ }^{1}$ Abnormal levels of $\mathrm{K}^{+}$ions in biological fluids can cause muscle cramps or weakness, nausea, diarrhoea, frequent urination, dehydration, paralysis, and changes in heart rhythms. $^{1}$

Potassium ions promote the formation of G-quadruplexed structures of telomeric DNA at the termini of the eukaryotic chromosome. The G-quadruplex of telomeric DNA is an unusual tetraplex conformation that has been demonstrated to directly inhibit telomerase activity in immortalized and most cancer cells. ${ }^{2,3}$ Thus, the ability to monitor the concentration of $\mathrm{K}^{+}$ions in biological fluids has become a very important challenge. Although many fluorescence indicators-including benzofuran isophthalate, coumarin diacid cryptand [2.2.2], pyrenyl-linked benzo[15]crown-5, and BODIPY-linked azacrown ethers and $\gamma$-cyclodextrin fluoroionophores - have been employed for the detection of $\mathrm{K}^{+}$ions, several drawbacks, such as the lack of selectivity against other alkali ions or the need for non-aqueous solutions, limit their clinical applications. ${ }^{4}$

Oligonucleotides have been used for their highly selective and sensitive detection to improve the accuracy of assays for $\mathrm{K}^{+}$ions. $^{5}$ Single-stranded (ss) and random-coiled oligonucleotides possessing four GGG sequences form uniquely selective $\mathrm{K}^{+}$binding sites in G-quadruplexes, ${ }^{5}$ presumably because of the similar sizes of the $\mathrm{K}^{+}$ion $(1.3 \AA)$ and the cavities created between two G-quadruplexes. ${ }^{5}$ Fluorescence resonance energy transfer (FRET) imaging has been used in conjunction with G-rich oligonucleotides possessing the human telomere sequence $\left[\mathrm{dG}_{3}\left(\mathrm{~T}_{2} \mathrm{AG}_{3}\right)_{3}\right]$ and the 15 -mer thrombin-binding aptamer, labeled with 6-carboxyfluorescein and 6carboxytetramethylrhodamine, respectively, to monitor $\mathrm{K}^{+}$ ions in an excess of $\mathrm{Na}^{+} .^{5 a, b}$ In addition, the $\mathrm{K}^{+}$-induced formation of efficiently emitting excimers from dGGTTGGTGTGGTTGG and pyrene has been demonstrated. ${ }^{5 c}$ Although cationic conjugated polymer-

Department of Chemistry, National Taiwan University, Taipei, Taiwan.E-mail: changht@ntu.edu.tw; Fax: (+886)-2-33661171; Tel: $(+886)-2-33661171$

† Electronic supplementary information (ESI) available: Experimental procedures on prepared compounds and Fig. S1-S7. See DOI: $10.1039 / \mathrm{b} 718752 \mathrm{a}$
G-quadruplex assemblies, G-quadruplex-induced aggregation of gold nanoparticles (Au NPs), and an electrochemical aptasensor have been developed for the selective detection of $\mathrm{K}^{+}$over $\mathrm{Na}^{+},{ }^{5 d-f}$ their applicability to biological systems is limited by the complicated nature of their sample matrices.

In this paper, we report a molecular fluorescence switching method to signal aptamer- $\mathrm{K}^{+}$binding using an ATP-binding aptamer (dACCTGGGGGAGTATTGCGGAGGAAGGT) and OliGreen (OG), a commercially available asymmetrical cyanine dye that is used to label oligonucleotides. ${ }^{6} \mathrm{OG}$ is weakly fluorescent, but exhibits a greater-than-1000-fold enhancement in its fluorescence upon binding to ssDNA (with excitation and emission maxima at 480 and $520 \mathrm{~nm}$, respectively). Scheme 1 outlines the sensing mechanism that we employed in this study. When the ATP-binding aptamer (Apt) in its random-coil (ss) structure is added to an OG solution, an OG.Apt complex is formed immediately, leading to increased fluorescence. After adding $\mathrm{K}^{+}$, the conformation of Apt changes from that of a random coil to two stacked Gquadruplexes and two short double-helix stems. Because OG prefers binding to ssDNA, dissociation of the OG.Apt complex occurs to some extent upon this conformational change. As a result, the fluorescence of the system decreases in the presence of $\mathrm{K}^{+}$.

Fig. 1 displays the fluorescence spectra that prove this concept. Curve A indicates that the fluorescence of $\mathrm{OG}$ $(0.025 X)$ in $5 \mathrm{mM}$ Tris- $\mathrm{HCl}(\mathrm{pH} 7.4)$ is very weak. In this study, we define the concentration of $\mathrm{OG}$ obtained from Molecular Probes (Portland, OR) as $100 X$. The fluorescence intensity at $520 \mathrm{~nm}$ of the OG solution $(0.025 X)$ increased by greater than 1000 -fold after the addition of $50 \mathrm{nM}$ randomcoil ATP Apt (curve B). We note that the fluorescence intensity reached a plateau at $50 \mathrm{nM}$ Apt when the concentration of OG was $0.125 X$ (Fig. S1, ESI $\dagger$ ). When $10 \mu \mathrm{M} \mathrm{K}^{+}$was added into a mixture of OG $(0.025 X)$ and Apt (50 nM), a rapid

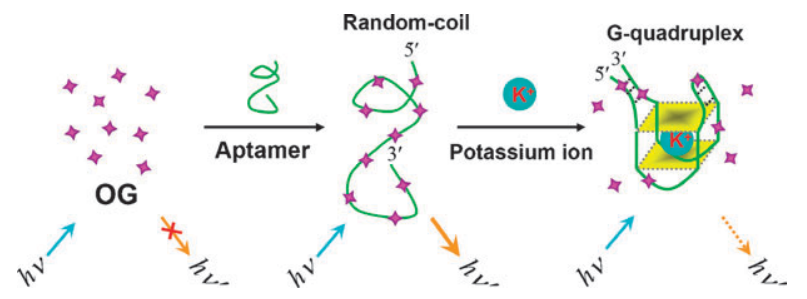

Scheme 1 Schematic representation of a $\mathrm{K}^{+}$sensor that operates based on modulation of the fluorescence of the complex formed between the dye OG and the ATP-binding aptamer. $h$ : Planck's constant; $\nu$ : frequency of light. 


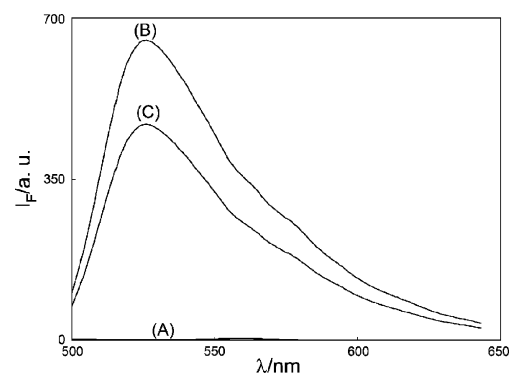

Fig. 1 Fluorescence spectra of solutions of (A) OG and (B, C) the OG.Apt complex in the (B) absence and (C) presence of $\mathrm{K}^{+}(10 \mu \mathrm{M})$. Solution: $10 \mathrm{mM}$ Tris- $\mathrm{HCl}$ (pH 7.4); excitation wavelength: $480 \mathrm{~nm}$. The fluorescence intensities $\left(I_{\mathrm{F}}\right)$ are plotted in arbitrary units $(\mathrm{au})$.

$(<3 \mathrm{~min})$ and significant decrease $(27 \%)$ in fluorescence intensity occurred (curve C), as would be expected upon the dissociation of the OG.Apt complex. To support our reasoning, we used centrifugal filters (molecular weigh cut-off: 1000 Da) to separate the dissociated OG from the OG.Apt complex. After labeling the dissociated OG with the oligonucleotide $\mathrm{dT}_{33}$ and measuring the fluorescence of the solution, we estimated that $c a .27 \%$ of the OG units had dissociated from the OG.Apt complex in the presence of $10 \mu \mathrm{M} \mathrm{K}^{+}$. To confirm that this behavior resulted from the binding of Apt and $\mathrm{K}^{+}$, we tested a control DNA strand having the sequence ACGTTAGCTACTATTCCGTACGAACCT that does not form a G-quadruplex structure to have a strong interaction with $\mathrm{K}^{+}$. In this case we did not observe any $\mathrm{K}^{+}(10 \mu \mathrm{M})$-induced changes in the fluorescence intensity of a mixture of $\mathrm{OG}$ $(0.025 X)$ and this DNA sample (data not shown).

We used capillary electrophoresis to investigate the stability of the G-quadruplex in the presence of $\mathrm{K}^{+}$(Fig. S2, ESI $\dagger$ ). In the absence of $\mathrm{K}^{+}$, we detected only the linear $\mathrm{OG} \cdot \mathrm{Apt}$ complex; in the presence of $\mathrm{K}^{+}$, we observed two signals corresponding to the linear and G-quadruplex OG.Apt complexes, respectively. ${ }^{7}$ Upon increasing the concentration of $\mathrm{K}^{+}$, the corrected peak area ratios of the linear $\mathrm{OG} \cdot \mathrm{Apt}$ complex decreased, while those of the G-quadruplex OG. Apt complex increased. This result suggests that the G-quadruplex OG.Apt complex was stable, both kinetically and thermodynamically, because of the tight association of the $\mathrm{K}^{+}$ions with the $\mathrm{G}$ residues.

We studied the effect of the OG concentration on the sensing of $10 \mu \mathrm{M} \mathrm{K}^{+}$in the presence of $50 \mathrm{nM} \mathrm{Apt.} \mathrm{The}$ value of $\left(I_{\mathrm{F} 0}-I_{\mathrm{F}}\right) / I_{\mathrm{F} 0}$ of the OG.Apt complex increased upon increasing the concentration of $\mathrm{OG}$ in the range $0.0001-0.01 X$, but it decreased at higher concentrations (Fig. S3, ESI $\dagger$ ) because of the higher fluorescence background. Next, we compared the effects of several DNA-binding dyes - ethidium bromide (EtBr), YOYO-3, TOTO-3, methylene blue, acridin orange, and $N, N$-dimethyl-2,7-diazapyrenium dication (DMDAP) - on the determination of $\mathrm{K}^{+}$using ATP Apt (50 $\mathrm{nM})$. We found that the presence of $\mathrm{K}^{+}$provided greater values of $\left(I_{\mathrm{F} 0}-I_{\mathrm{F}}\right) / I_{\mathrm{F} 0}$ for the OG.Apt complex than it did for the other DNA-binding dye-Apt complexes (Fig. S4, ESI $\dagger$ ). Because EtBr, YOYO-3, and TOTO-3 are double-strandspecific DNA-binding dyes, ${ }^{8}$ we suspect that the presence of $\mathrm{K}^{+}$did not induce the dissociation of those dyes as well as it did $\mathrm{OG}$ from their respective complexes. Because methylene blue, acridin orange, and DMDAP all have weaker binding affinities toward DNA $\left(K_{\mathrm{a}}=10^{4}-10^{6} \mathrm{M}^{-1}\right)$ than does $\mathrm{OG}{ }^{9}$ their sensitivity for the detection of $\mathrm{K}^{+}$was lower than that of $\mathrm{OG}$ in the presence of Apt. We conclude that, among the complexes we tested, the OG.Apt complex was the most sensitive toward $\mathrm{K}^{+}$ions.

Fig. 2(a) depicts the changes in fluorescence of the OG.Apt complex upon the addition of $\mathrm{K}^{+}(0-5.0 \mu \mathrm{M})$ under the optimum conditions (0.01X OG, $50 \mathrm{nM}$ ATP Apt, $10 \mathrm{mM}$ Tris-HCl, pH 7.4). The OG.Apt sensor exhibited a linear increase in its value of $\left(I_{\mathrm{F} 0}-I_{\mathrm{F}}\right) / I_{\mathrm{F} 0}$ upon increasing the $\mathrm{K}^{+}$concentration from 100 to $1000 \mathrm{nM}$. The limit of detection (LOD) was found to be $75 \mathrm{nM} \mathrm{K}{ }^{+}$, based on a signal-to-noise ratio $(\mathrm{S} / \mathrm{N})$ of 3 . Thus, our approach pushes the sensitivity lower, by one order of magnitude, relative to those of other reported G-quadruplex-mediated $\mathrm{K}^{+}$sensors. ${ }^{5}$ Although the linear range of our probe is narrow, it can be tuned by changing the concentrations of OliGreen and Apt. When using higher concentrations of OliGreen and Apt, we can achieve a wider linear range despite having a lower sensitivity. For example, we can achieve a linear range 0.25-5.0 $\mu \mathrm{M}$ (LOD $0.22 \mu \mathrm{M}$ ) when using $0.05 X$ OliGreen and $250 \mathrm{nM}$ Apt. The binding constant $\left(K_{\mathrm{b}}\right)$ for the interaction between the OG.Apt complex and $\mathrm{K}^{+}$ions, calculated (by fitting experimental data to an equation derived for a complex having $1: 1$ stoichiometry) from the plot provided in the inset to Fig. 2, was $2.5 \times$ $10^{6} \mathrm{M}^{-1}$ (Fig. S5, ESI $\dagger$ ). ${ }^{5 b}$ The ultralow background fluorescence of $\mathrm{OG}$ and the high affinity of Apt toward $\mathrm{K}^{+}$are two factors contributing to this system's greater sensitivity relative to that of traditional sensors. ${ }^{5}$ The $\mathrm{K}^{+}$binding oligonucleotide possessing the human telomere sequence $\left[\mathrm{dG}_{3}\left(\mathrm{~T}_{2} \mathrm{AG}_{3}\right)_{3}\right]$ was also tested for $\mathrm{K}^{+}$sensing (Fig. S6, ESI $\dagger$ ). The linear relationship exhibited increases in the value of $\left(I_{\mathrm{F} 0}-I_{\mathrm{F}}\right) / I_{\mathrm{F} 0}$ upon increasing the $\mathrm{K}^{+}$concentration over the range 10 $250 \mu \mathrm{M}$. The limit of detection (LOD) was $8.5 \mu \mathrm{M}$. However,
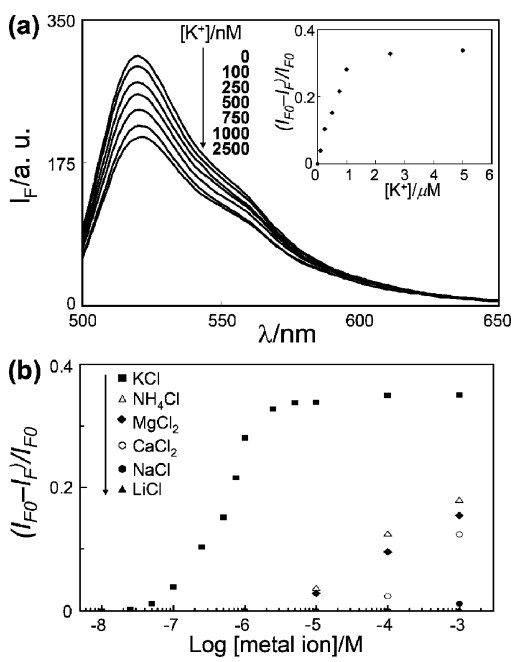

Fig. 2 (a) Fluorescence response of the OG·Apt complex (50 nM) upon addition of $\mathrm{K}^{+}$ions. (b) Plot of the fluorescence signal decrease ratio $\left(I_{\mathrm{F} 0}-I_{\mathrm{F}} / I_{\mathrm{F} 0}\right)$ of the OG.Apt complex at $520 \mathrm{~nm}$ against the concentration of $\mathrm{KCl}, \mathrm{NH}_{4} \mathrm{Cl}, \mathrm{MgCl}_{2}, \mathrm{CaCl}_{2}, \mathrm{NaCl}$, and LiCl. $I_{\mathrm{F} 0}$ and $I_{\mathrm{F}}$ are the fluorescence intensities of the OG.Apt complex in the absence and presence of the additional ions, respectively. 
Table 1 Concentrations of $\mathrm{K}^{+}$in urine samples from seven healthy volunteers measured using the OG·Apt complex fluorescence sensor ${ }^{a}$ and ICP-MS

\begin{tabular}{llll}
\hline & $\begin{array}{l}\text { OG·Apt sensor } \\
\text { Mean } \pm \text { SD/ } \\
\mathrm{mM}(n=5)\end{array}$ & $\begin{array}{l}\text { ICP-MS } \\
\text { Mean } \pm \mathrm{SD} / \\
\mathrm{mM}(n=5)\end{array}$ & $\begin{array}{l}\text { F-test between } \\
\text { two methods }^{b}\end{array}$ \\
\hline $\mathrm{A}$ & $15.0 \pm 1.5$ & $16.0 \pm 1.8$ & 1.44 \\
$\mathrm{~B}$ & $44.4 \pm 2.6$ & $45.3 \pm 3.2$ & 1.51 \\
$\mathrm{C}$ & $53.1 \pm 2.1$ & $56.2 \pm 4.3$ & 4.19 \\
$\mathrm{D}$ & $15.4 \pm 1.9$ & $16.5 \pm 2.1$ & 1.22 \\
$\mathrm{E}$ & $20.6 \pm 2.0$ & $21.5 \pm 1.2$ & 2.78 \\
$\mathrm{~F}$ & $48.3 \pm 2.5$ & $51.3 \pm 3.2$ & 1.64 \\
$\mathrm{G}$ & $29.3 \pm 2.3$ & $32.3 \pm 1.3$ & 5.29
\end{tabular}

${ }^{a} 50 \mathrm{nM} .{ }^{b}$ The F-test value is 6.39 at a $95 \%$ confidence level.

its sensitivity is lower than that on using ATP aptamer, mainly because the formation constant for the $\mathrm{K}^{+}$-aptamer complex is smaller than that of $\mathrm{K}^{+}-\mathrm{ATP}$ aptamer complex. ${ }^{5}$

We investigated the selectivity of our new sensor toward $\mathrm{K}^{+}$ over other ions, namely $\mathrm{Na}^{+}, \mathrm{Li}^{+}, \mathrm{NH}_{4}{ }^{+}, \mathrm{Mg}^{2+}$, and $\mathrm{Ca}^{2+}$, under the optimum conditions. When we plotted the values of $\left(I_{\mathrm{F} 0}-I_{\mathrm{F}}\right) / I_{\mathrm{F} 0}$ for the OG.Apt complex against the concentration of the tested ions (Fig. 2b), we found that only $\mathrm{K}^{+}$ provided a significant positive response; only when the concentrations of $\mathrm{NH}_{4}^{+}, \mathrm{Mg}^{2+}, \mathrm{Ca}^{2+}$, and $\mathrm{Na}^{+}$exceeded 0.01 , $0.01,0.10$, and $1.00 \mathrm{mM}$, respectively, did these ions induce slight dissociations of the OG.Apt complex. Generally, the ionic radius is the parameter that determines how well G-quadruplex structures are stabilized by various cations. ${ }^{2,5}$ The stabilities of alkali and alkali earth metal ions in Gquadruplex structures decrease in the orders $\mathrm{K}^{+} \gg \mathrm{Na}^{+}>$ $\mathrm{Rb}^{+}>\mathrm{Cs}^{+}>\mathrm{Li}^{+}$and $\mathrm{Sr}^{2+} \gg \mathrm{Ba}^{2+}>\mathrm{Ca}^{2+}>\mathrm{Mg}^{2+}$, respectively. ${ }^{5}$ The selectivity of our OG.Apt complex sensor toward $\mathrm{K}^{+}$over $\mathrm{Na}^{+}$was greater than 10000 -fold, which is much higher than the values obtained for the potassiumbinding benzofuran isophthalate (1.5) and coumarin diacid cryptand [2.2.2] (3.4) and is comparable to that of the best reported G-quadruplex-based $\mathrm{K}^{+}$sensor. ${ }^{4,5}$ Note, however, that our simple method does not require covalent labeling of Apt prior to analysis.

We tested our OG·Apt complex sensor for the determination of $\mathrm{K}^{+}$ions under physiological conditions. Aliquots of $\mathrm{K}^{+}(0-20 \mathrm{mM})$ were spiked into synthetic solutions $(145 \mathrm{mM}$ $\mathrm{Na}^{+}, 1.5 \mathrm{mM} \mathrm{Mg}{ }^{2+}$, and $2.5 \mathrm{mM} \mathrm{Ca}^{2+}$ ) and then the mixtures were diluted with $10 \mathrm{mM}$ Tris- $\mathrm{HCl}(\mathrm{pH}$ 7.4). The value of $\left(I_{\mathrm{F} 0}-I_{\mathrm{F}}\right) / I_{\mathrm{F} 0}$ of the OG.Apt complex increased linearly upon increasing the concentration of spiked $\mathrm{K}^{+}$ions from 0 to $10 \mathrm{mM}$ (Fig. S7, ESI $\dagger$ ); the recovery of $\mathrm{K}^{+}$was 93-98\%. Next, we tested the practicality of this present approach for the analysis of $\mathrm{K}^{+}$ions in urine samples. The level of $\mathrm{K}^{+}$in urine is indicative of certain kidney diseases. ${ }^{10}$ The normal range of $\mathrm{K}^{+}$excreted from a healthy human being on a regular diet is $25-125 \mathrm{mmol} \mathrm{L}^{-1}$ per $24 \mathrm{~h}$. We filtered urine samples from seven healthy volunteers through $0.2 \mu \mathrm{m}$ membranes, diluted the samples 100000 -fold using $10 \mathrm{mM}$ Tris- $\mathrm{HCl}(\mathrm{pH} 7.4)$, and then analyzed them using the $50 \mathrm{nM}$ OG.Apt complex. Table 1 lists the results obtained using both our sensor and inductively coupled plasma mass spectrometry (ICP-MS). On the basis of an F-test, the results from our present approach are in good agreement with those obtained using ICP-MS. Note that, when using our OG.Apt complex sensor, the sample preparation and analysis time was less than $15 \mathrm{~min}$; this assay also provides the advantages of simplicity and cost-effectiveness, and it is amenable to high throughput.

In conclusion, we have developed a homogenous $\mathrm{K}^{+}$assay using ATP Apt and OG. Upon binding to $\mathrm{K}^{+}$ions, the OG. Apt complex having a linear structure converts into a G-quadruplex structure. As a result, some of the OG units dissociate from the complex, leading to a decrease in fluorescence. The selectivity of this system for $\mathrm{K}^{+}$over other ions in aqueous solution is remarkably high $\left(>10000\right.$-fold over $\mathrm{Na}^{+}$) and its LOD is $75 \mathrm{nM}$. The present approach has several advantages over other assays: it is rapid ( $<15 \mathrm{~min})$, label-free, simple, and cost-effective. This sensing strategy is not limited to probing the formation of G-quadruplexes and the detection of $\mathrm{K}^{+}$: it can be applied to investigations of the conformational changes that occur to most biomolecules upon binding to their targets.

We are grateful to the National Science Council of Taiwan for providing financial support for this study under contract NSC 95-2113-002-026-MY3. C.-C. Huang thanks National Taiwan University for PDF support.

\section{Notes and references}

1 (a) P. Kofuji and E. A. Newman, Neuroscience, 2004, 129, 1045-1056; (b) P. M. Suter, Nutr. Rev., 1998, 56, 151-153; (c) S. F. Pedersen, M. E. O'Donnell, S. E. Anderson and P. M. Cala, Am. J. Physiol.: Regul., Integr. Comp. Physiol., 2006, 291, R1-R25.

2 (a) M. Gellert, M. N. Lipsett and D. R. Davies, Proc. Natl. Acad. Sci. U. S. A., 1962, 48, 2013-2018; (b) M. Lu, Q. Guo and N. R. Kallenbach, Biochemistry, 1993, 32, 598-601.

3 (a) A. M. Zahler, J. R. Williamson, T. R. Cech and D. M. Prescott, Nature, 1991, 350, 718-720; (b) S. Neidle and G. Parkinson, Nat. Rev. Drug Discovery, 2002, 1, 383-393.

4 (a) K. Meuwis, N. Boens, F. C. De Schryver, J. Gallay and M. Vincent, Biophys. J., 1995, 68, 2469-2473; (b) P. Buet, B. Gersch and E. Grell, J. Fluoresc., 2001, 11, 79-87; (c) A. Yamauchi, T. Hayashita, S. Nishizawa, M. Watanabe and N. Teramae, J. Am. Chem. Soc., 1999, 121, 2319-2320; (d) M. Baruah, W. Qin, R. A. L. Vallée, D. Beljonne, T. Rohand, W. Dehaen and N. Boens, Org. Lett., 2005, 7, 4377-4380; (e) A. Yamauchi, T. Hayashita, A. Kato, S. Nishizawa, M. Watanabe and N. Teramae, Anal. Chem., 2000, 72, 5841-5846.

5 (a) B. Juskowiak, Anal. Chim. Acta, 2006, 568, 171-180; (b) S. Nagatoishi, T. Nojima, E. Galezowskab, A. Gluszynska, B. Juskowiak and S. Takenaka, Anal. Chim. Acta, 2007, 581, 125-131; (c) S. Nagatoishi, T. Nojima, B. Juskowiak and S. Takenaka, Angew. Chem., Int. Ed., 2005, 44, 5067-5070; (d) F. He, Y. Tang, S. Wang, Y. Li and D. Zhu, J. Am. Chem. Soc., 2005, 127, 12343-12346; (e) L. Wang, X. Liu, X. Hu, S. Song and C. Fan, Chem. Commun., 2006, 3780-3782; (f) A.-E. Radi and C. K. O'Sullivan, Chem. Commun., 2006, 3432-3434.

6 R. P. Haugland, in The Handbook-A Guide to Fluorescent Probes and Labeling Technologies, ed. B. M. T. Z. Spence, Invitrogen Corp., Carlsbad, CA, 10th edn, 2005, ch. 8, pp. 357-358.

7 C.-C. Huang, Z. Cao, H.-T. Chang and W. Tan, Anal. Chem., 2004, 76, 6973-6981.

8 (a) M. Mecklenburg, A. Grauers, B. R. Jönsson, A. Weber and B. Danielsson, Anal. Chim. Acta, 1997, 347, 79-86; (b) M. J. Joseph, J. C. Taylor, L. B. McGown, J. B. Pitner and C. P. Linn, Biospectroscopy, 1996, 2, 173-183.

9 (a) S. Nafisi, A. A. Saboury, N. Keramat, J.-F. Neault and H.-A. Tajmir-Riahi, J. Mol. Struct., 2007, 827, 35-43; (b) C.-C. Huang, S.-H. Chiu, Y.-F. Huang and H.-T. Chang, Anal. Chem., 2007, 79, 4798-4804; (c) H.-C. Becker and B. Nordén, J. Am. Chem. Soc., 1997, 119, 5798-5803.

10 R. L. Jamison, Kidney Int., 1987, 31, 695-703. 